\title{
AFTERWORD TO THE ARTICLE "A BRIEF HISTORY OF THE CLASSIFICATION OF THE FINITE SIMPLE GROUPS"
}

\author{
RON SOLOMON
}

\begin{abstract}
In this afterword the author discusses his previously published article "A brief history of the classification of the finite simple groups", which appeared in Bull. Amer. Math. Soc. (N.S) 38 (2001), no. 3, 315-352.
\end{abstract}

Shortly after the publication of my article about the classification of finite simple groups (CFSG), I received a phone call from Serge Lang, who chided me in his inimitable fashion for asserting that the Quasithin Theorem of Aschbacher and Smith would probably be published in 2001 or 2002. You can never assert that a theorem will be proved until it is proved, said Serge, and of course he was right. I am happy to report that my optimism in this case was well founded, though the actual publication date for the Aschbacher-Smith monographs [3] was 2004.

The revision projects discussed in Section 9 of that article have continued and indeed multiplied. The GLS (Gorenstein, Lyons, and Solomon) Project has produced seven volumes thus far, and it is discussed in some detail in my recent Notices article [28. So I shall say little more about the status of that project here.

The GLS Project aims to identify the finite simple groups from their $p$-local structure, when the $p$-elements of $G$ are semisimple, i.e., the centralizers of some $p$-elements are reductive groups. In contrast, the MSS Project, led by Meierfrankenfeld, Stellmacher, and Stroth 21] aims to recognize $G$ from its $p$-local structure, where $p$ is a unipotent prime divisor of $|G|$, i.e., the maximal $p$-local subgroups of $G$ are parabolic subgroups. One of the principal achievements of the MSS Project to date is the proof [22] of the Local Structure Theorem for finite groups $G$ with a large $p$-subgroup $Q$ (in the sense that $C_{G}(Q)=Z(Q)$ and $N_{G}(U) \leq N_{G}(Q)$ for all $1 \leq U \leq Z(Q))$. They identify the structure of most of the maximal parabolic subgroups of these groups. (I write here as if $G$ is known to be of Lie type, which of course is not the case. Nevertheless this language can be justified in full generality. See [28] for details.)

From this data, it is still necessary to identify $G$, and in this direction, Meierfrankenfeld, Stroth, and Weiss have proved such an identification theorem in 23. Along the way, they have given a short proof of the uniqueness of spherical buildings of rank at least 3 in the finite case. This, of course, carries on a theme originating with Jacques Tits. A theorem of Curtis and Tits, as well as related theorems of Phan, provides the key tool for the GLS identification of simple groups of Lie type from their semisimple data. Under the general heading of Phan Theory, this subject has been expanded and clarified in recent work by many authors, starting

Received by the editors July 2, 2018.

2010 Mathematics Subject Classification. Primary 20D05. 
with a paper of Bennett and Shpectorov 4 and culminating in a paper of Blok, Hoffman, and Shpectorov [5. The considerable body of intervening work is ably described by Gramlich in [12].

The identification of simple groups of characteristic $p$-type from their unipotent structure dates back to work of John G. Thompson on group factorizations and failure of factorization. The impediments to factorization were dubbed offender, and recent work of Meierfrankenfeld, Parmeggianni, and Stellmacher [19] provides a general offender theory unifying and extending much of this work.

If completed (pace Serge Lang), the GLS and MSS Projects will provide two almost completely different approaches to the identification of finite simple groups those of even type (or local characteristic 2). The GLS Project also treats the other finite simple groups, i.e., those of odd type, mainly the alternating groups and the groups of Lie type defined over a finite field of odd order. An alternative approach to these groups, led by Aschbacher, proceeds in the category of (saturated) fusion systems defined over a finite 2-group. The notion of a fusion system (or Frobenius category) was first introduced by Puig, and it was studied extensively by Bob Oliver with numerous collaborators. For Oliver, the original goal was a proof [25, 26] of the Martino-Priddy Conjecture that two finite groups have isomorphic $p$-fusion categories if and only if their $p$-completed classifying spaces are homotopy equivalent. A key step was the proof (using CFSG) by Oliver that every fusion system for a finite group has a unique associated centric linking system. A new approach yielding a major generalization of this result was provided by Andy Chermak [7, again using CFSG. Glauberman and Lynd 11 then showed how Chermak's proof could be made independent of the CFSG.

Aschbacher in [1] and 2] has spearheaded an effort to provide a classification of all simple fusion systems based on a finite 2 -group $S$. The working conjecture is that every such abstract 2-fusion system arises as the 2-fusion system of a finite simple group $G$ with Sylow 2-subgroup $S$, except for one infinite family, sometimes called the Benson-Solomon 2-fusion systems. Like the classification problem for finite simple groups, this problem subdivides naturally into the case of even systems and the case of odd systems. The former case can be treated by methods almost identical to those of the MSS Project. Aschbacher has focused on the odd systems, and has made substantial progress toward the completion of this case. This would not replace the GLS treatment of simple groups of odd type, but it would independently classify all possible 2-fusion systems for such groups (with the exception of the socalled Goldschmidt groups). Thus, if the GLS, MSS, and Aschbacher Projects all come to fruition, we will be in the enviable position of having two substantially different treatments of the large majority of the Classification proof. (Like the Cheese in the nursery rhyme, the Odd Order Paper of Feit and Thompson still stands alone.)

Of course, a major justification for this belt-and-suspenders approach to the Classification proof is the large number of significant consequences of the CFSG, both within and outside finite group theory. We mention just a few. A recent book by Steve Smith [27] describes many more. (Also, see [30.) Within finite group theory, the Schreier Conjecture (the outer automorphism group of every simple group is solvable) is an easy consequence of the CFSG and Steinberg's work on automorphisms of groups of Lie type. The finite 2-transitive permutation groups are now classified. (See 6.) We now know that every finite simple group can be 
generated by two elements, one of which can be fixed. (Without the CFSG, there is no known bound.) Moreover, the probability that two random elements generate a finite simple group goes to 1 as the group order goes to $\infty$. The program to determine the maximal subgroups of the finite simple groups (equivalently, their primitive permutation representations), initiated by O'Nan and Len Scott, has made great progress thanks to work of Aschbacher, Liebeck, Seitz, Testerman, and others.

In the domain of representation theory, some significant breakthroughs have been achieved recently using CFSG and the deep theorems of Lusztig and others. Many years ago, John McKay observed that for many simple groups $G$, the number of ordinary irreducible characters of odd degree for both $G$ and $N_{G}(S)$ were the same, where $S$ is a Sylow 2-subgroup of $G$. He conjectured that this was always the case. This McKay Conjecture (for all finite groups) has recently been proved by Malle and Späth 20. It is the tip of an iceberg of conjectures - the Alperin-McKay Conjecture, the Alperin Weight Conjecture, and variations thereon by Dade, Isaacs, Navarro, Turull, and others. General reduction theorems have been developed by numerous authors, notably Isaacs, Malle, and Navarro [16] and Späth [29], reducing these conjectures to related conjectures about the representation theory and cohomology of finite simple groups.

Yet another conjecture, the Brauer Height Zero Conjecture, has been proved for all finite quasi-simple groups (and, in one direction, for all finite groups) by Kessar and Malle [17, 18. Thanks to work of Navarro and Späth [24, the full conjecture will follow for all finite groups from the inductive form of the Alperin-McKay Conjecture for finite simple groups. All of these results use the CFSG.

My thanks to Bob Guralnick for providing the following sampling of applications of the CFSG to other areas. One of the first and still one of the nicest consequences is the fact that any finite transitive permutation group of degree $n>1$ contains an element of prime power order which has no fixed points. (If one drops the prime power order condition, this is a very elementary theorem of Camille Jordan.) This was originally stated in [8] and generalized in various ways (cf., [10]). What is surprising about this result is that it is equivalent to a fundamental result in number theory which asserts that if $L / K$ is a nontrivial extension of number fields, then the relative Brauer group of $L / K$ is infinite, i.e., there are infinitely many nonisomorphic finite-dimensional division algebras $D$ with center $K$ such that $D \otimes_{K} L$ is a matrix algebra over $L$. Remarkably, these two results are equivalent.

A polynomial $f(x)$ over a finite field is called exceptional if it becomes bijective over some sufficiently large extension field. These were introduced by Dickson in the 1890s, and variations of them were studied by Schur. Using the CFSG, almost all indecomposable exceptional polynomials have been classified [13, 15]. This involved classifying certain types of permutation groups [9] and then determining when there were polynomials attached to the groups, and finding all of them.

In his thesis Zariski answered a conjecture of Enriques by showing that a generic Riemann surface of genus greater than 6 has no solvable map to the Riemann sphere. This was generalized in a series of papers (the latest being [14]) to show that if there is a degree $n$ indecomposable map from a generic Riemann surface of genus $g \geq 4$, then the associated monodromy group is either the alternating group of degree $n$ with $n>2 g$ or the symmetric group of degree $n$ with $n>\frac{g+1}{2}$. 
Thus, the future study of finite groups, their representations, and their applications remains as vibrant as was its history.

\section{REFERENCES}

[1] M. Aschbacher, R. Kessar, and B. Oliver, Fusion systems in algebra and topology, London Mathematical Society Lecture Note Series, vol. 391, Cambridge University Press, Cambridge, 2011. MR2848834

[2] M. Aschbacher and B. Oliver, Fusion systems, Bull. Amer. Math. Soc. (N.S.) 53 (2016), no. 4, 555-615, DOI 10.1090/bull/1538. MR3544261

[3] M. Aschbacher and S. D. Smith, The classification of quasithin groups. I: Structure of strongly quasithin K-groups, Mathematical Surveys and Monographs, vol. 111, American Mathematical Society, Providence, RI, 2004. MR2097623

[4] C. D. Bennett and S. Shpectorov, A new proof of a theorem of Phan, J. Group Theory 7 (2004), no. 3, 287-310, DOI 10.1515/jgth.2004.010. MR2062999

[5] R. Blok, C. Hoffman and S. Shpectorov, Classification of Curtis-Tits and Phan amalgams with 3-spherical diagram, Israel J. Math. (to appear).

[6] P. J. Cameron, Finite permutation groups and finite simple groups, Bull. London Math. Soc. 13 (1981), no. 1, 1-22, DOI 10.1112/blms/13.1.1. MR599634

[7] A. Chermak, Fusion systems and localities, Acta Math. 211 (2013), no. 1, 47-139, DOI 10.1007/s11511-013-0099-5. MR.3118305

[8] B. Fein, W. M. Kantor, and M. Schacher, Relative Brauer groups. II, J. Reine Angew. Math. 328 (1981), 39-57. MR636194

[9] M. D. Fried, R. Guralnick, and J. Saxl, Schur covers and Carlitz's conjecture, Israel J. Math. 82 (1993), no. 1-3, 157-225, DOI 10.1007/BF02808112. MR1239049

[10] R. M. Guralnick, Zeroes of permutation characters with applications to prime splitting and Brauer groups, J. Algebra 131 (1990), no. 1, 294-302, DOI 10.1016/0021-8693(90)90177-P. MR:1055010

[11] G. Glauberman and J. Lynd, Control of fixed points and existence and uniqueness of centric linking systems, Invent. Math. 206 (2016), no. 2, 441-484, DOI 10.1007/s00222-016-0657-5. MR 3570297

[12] R. Gramlich, Developments in finite Phan theory, Innov. Incidence Geom. 9 (2009), 123-175. MR2658896

[13] R. M. Guralnick, J. Rosenberg, and M. E. Zieve, A new family of exceptional polynomials in characteristic two, Ann. of Math. (2) 172 (2010), no. 2, 1361-1390, DOI 10.4007/annals.2010.172.1367. MR2680493

[14] R. M. Guralnick and J. Shareshian, Symmetric and alternating groups as monodromy groups of Riemann surfaces. I. Generic covers and covers with many branch points, Mem. Amer. Math. Soc. 189 (2007), no. 886, vi+128, DOI 10.1090/memo/0886. With an appendix by Guralnick and R. Stafford. MR2343794

[15] R. M. Guralnick and M. E. Zieve, Polynomials with PSL(2) monodromy, Ann. of Math. (2) 172 (2010), no. 2, 1315-1359, DOI 10.4007/annals.2010.172.1321. MR2680492

[16] I. M. Isaacs, G. Malle, and G. Navarro, A reduction theorem for the McKay conjecture, Invent. Math. 170 (2007), no. 1, 33-101, DOI 10.1007/s00222-007-0057-y. MR2336079

[17] R. Kessar and G. Malle, Quasi-isolated blocks and Brauer's height zero conjecture, Ann. of Math. (2) 178 (2013), no. 1, 321-384, DOI 10.4007/annals.2013.178.1.6. MR3043583

[18] R. Kessar and G. Malle, Brauer's height zero conjecture for quasi-simple groups, J. Algebra 475 (2017), 43-60, DOI 10.1016/j.jalgebra.2016.05.010. MR3612463

[19] U. Meierfrankenfeld, G. Parmeggiani, and B. Stellmacher, General offender theory, J. Algebra 495 (2018), 264-288, DOI 10.1016/j.jalgebra.2017.11.005. MR.3726111

[20] G. Malle and B. Späth, Characters of odd degree, Ann. of Math. (2) 184 (2016), no. 3, 869-908, DOI 10.4007/annals.2016.184.3.6. MR3549625

[21] U. Meierfrankenfeld, B. Stellmacher, and G. Stroth, Finite groups of local characteristic $p$ : an overview, Groups, combinatorics \& geometry (Durham, 2001), World Sci. Publ., River Edge, NJ, 2003, pp. 155-192, DOI 10.1142/9789812564481_0010. MR1994966

[22] U. Meierfrankenfeld, B. Stellmacher, and G. Stroth, The local structure for finite groups with a large p-subgroup, Mem. Amer. Math. Soc. 242 (2016), no. 1147, vii+342, DOI 10.1090/memo/1147. MR 3517155 
[23] U. Meierfrankenfeld, G. Stroth, and R. M. Weiss, Local identification of spherical buildings and finite simple groups of Lie type, Math. Proc. Cambridge Philos. Soc. 154 (2013), no. 3, 527-547, DOI 10.1017/S0305004113000078. MR3044213

[24] G. Navarro and B. Späth, On Brauer's height zero conjecture, J. Eur. Math. Soc. (JEMS) 16 (2014), no. 4, 695-747, DOI 10.4171/JEMS/444. MR3191974

[25] B. Oliver, Equivalences of classifying spaces completed at odd primes, Math. Proc. Cambridge Philos. Soc. 137 (2004), no. 2, 321-347, DOI 10.1017/S0305004104007728. MR2092063

[26] B. Oliver, Equivalences of classifying spaces completed at the prime two, Mem. Amer. Math. Soc. 180 (2006), no. 848, vi+102, DOI 10.1090/memo/0848. MR2203209

[27] S. D. Smith, Applying the classification of finite simple groups: A user's guide, Mathematical Surveys and Monographs, vol. 230, American Mathematical Society, Providence, RI, 2018. MR.3753581

[28] R. Solomon, The classification of finite simple groups: a progress report, Notices Amer. Math. Soc. 65 (2018), no. 6, 646-651.

[29] B. Späth, A reduction theorem for the Alperin-McKay conjecture, J. Reine Angew. Math. 680 (2013), 153-189, DOI 10.1515/crelle.2012.035. MR3100954

[30] P. H. Tiep, Representation of finite groups: conjectures, reductions, and applications, Acta Math. Vietnam. 39 (2014), no. 1, 87-109, DOI 10.1007/s40306-013-0043-y. MR3176464

Department of Mathematics, Ohio State University, Columbus, Ohio

Email address: solomon@math.ohio-state.edu 